\title{
AIRBED: A Simplified Density Functional Theory Model for Physisorption on Surfaces
}

\author{
Stephen E. Mason ${ }^{\dagger}$, Peter H. Beton ${ }^{\ddagger}$ and Nicholas A. Besley ${ }^{\dagger *}$ \\ ${ }^{\ddagger}$ School of Physics \& Astronomy, University of Nottingham, University Park, Nottingham \\ $N G 7$ 2RD, UK. \\ ${ }^{\dagger}$ School of Chemistry, University of Nottingham, University Park, Nottingham, NG7 2RD, \\ $U K$. \\ E-mail: nick.besley@nottingham.ac.uk
}

\begin{abstract}
Dispersion interactions are commonly included in density functional theory (DFT) calculations through the addition of an empirical correction. In this study, a modification is made to the damping function in DFT-D2 calculations, to describe repulsion at small internuclear distances. The resulting Atomic Interactions Represented By Empirical Dispersion (AIRBED) approach is used to model the physisorption of molecules on surfaces such as graphene and hexagonal boron nitride, where the constituent atoms of the surface are no longer required to be included explicitly in the density functional theory calculation but are represented by a point charge to capture electrostatic effects. It is shown that this model can reproduce the structures predicted by full DFT-D2 calculations to a high degree of accuracy. The significant reduction in computational cost allows much larger systems to be studied, including molecular arrays on surfaces and sandwich complexes involving organic molecules between two surface layers.
\end{abstract}


Table of Contents Graphic

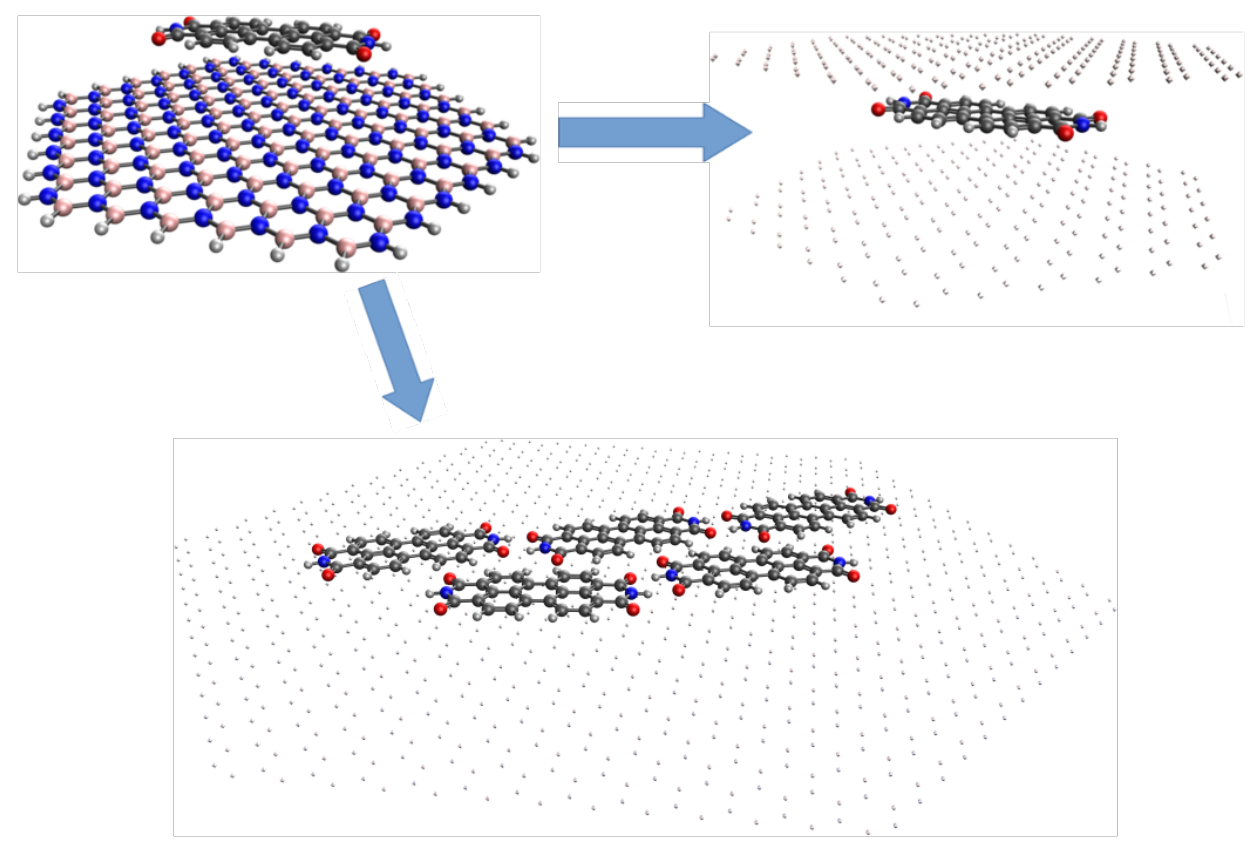




\section{Introduction}

The study of 2-dimensional (2D) arrays of organic molecules on surfaces is an increasingly important area of research owing to their potential applications in a range of fields including electronic and optoelectronic devices. ${ }^{1-4}$ Understanding the interactions that underpin the organisation of these arrays is key to realising their potential. These interactions can include hydrogen bonding, covalent bonding, dispersion forces and metal coordination, which may all have a significant role in the structure of the array. ${ }^{5-8}$ One area of particular focus is the study of organic arrays on surfaces such as graphene and hexagonal boron nitride $(\mathrm{hBN})$. These arrays can be imaged using scanning tunnelling microscopy and atomic force microscopy, which can provide high-resolution images of 2D molecular and supramolecular organization under ultra-high-vacuum conditions and at atmospheric pressure. ${ }^{9-13}$ When adsorbed on insulating surfaces, the fluorescence of the adsorbed molecular layers can also be measured providing a direct link to the optoelectronic properties of the arrays. ${ }^{14-20}$

Recent experimental work has shown shifts in the fluorescence emission bands arising from adsorption on $\mathrm{hBN}$ can be measured, and it has also been demonstrated that these shifts can arise from changes in the molecular structure that occur on absorption, and other

factors such as screening effects that depend on the refractive index of the substrate. ${ }^{14-20}$ Quantum chemical calculations, for example density functional theory (DFT), can make a significant contribution to characterising the structure of adsorbed molecular arrays and also their optoelectronic properties. An inherent limitation of molecular quantum chemical calculations for these systems is the computational cost of treating a large organic molecule and the model of the surface so that only a single molecule may be typically considered. ${ }^{18}$ Clearly it is desirable that many adsorbed organic molecules are included in the calculation to capture the effects of the larger molecular array, and this motivates the search for computationally less expensive approaches that include the important interactions that can be used to model the structure of these systems. 
Quantum chemical methods, including coupled cluster theory, have been used to determine the absorption energies of small molecules on molecular models of the graphene and fluorographene surfaces. ${ }^{21-23}$ A symmetry adapted perturbation theory analysis showed that the dominant contribution to the adsorption energy arises from dispersion ${ }^{21}$ which is consistent with other work which also found that dispersion is the most significant interaction between the insulating surface and adsorbed array for systems where the molecular arrays are physisorbed on the surface. ${ }^{18}$ The next largest contribution to the adsorption energy came from the electrostatic interactions. In this work we focus on DFT since this can be most readily applied to larger systems. Incorporating dispersion forces within DFT is an active area of research. ${ }^{24-33}$ However, the most computationally efficient approaches are empirical dispersion models. An example of this approach is the DFT-D2 dispersion correction of Grimme, ${ }^{28}$ in this approach the total energy is expressed as

$$
E_{D F T-D 2}=E_{K S-D F T}+E_{D I S P}
$$

where $E_{K S-D F T}$ is the usual DFT energy according to the chosen functional, and $E_{D I S P}$ is the dispersion energy that is typically given by

$$
\begin{aligned}
E_{D I S P} & =-s_{6} \sum_{A}^{N} \sum_{B<A}^{N} \frac{C_{6}^{A B}}{R_{A B}^{6}} f_{d m p}\left(R_{A B}\right) \\
C_{6}^{A B} & =\left(C_{6}^{A} C_{6}^{B}\right)^{1 / 2} \\
f_{d m p}\left(R_{A B}\right) & =\left[1+e^{-d\left(R_{A B} / R_{A B}^{0}-1\right)}\right]^{-1} .
\end{aligned}
$$

Here $R_{A B}$ and $R_{A B}^{0}$ are the internuclear separation and sum of the van der Waals radii of atoms $A$ and $B$ respectively, $C_{6}^{A B}$ is the dispersion coefficient for atom pair $A B, s_{6}$ is a scaling factor and there are $N$ atoms in the system. The role of the damping function, $f_{d m p}\left(R_{A B}\right)$ is to avoid double counting of electron correlation effects and the near-singularites as $R_{A B} \rightarrow 0$. In this paper, we describe a modification to the damping function so that it also describes the 
Pauli repulsion between the atom pairs, with the contribution from electrostatic interactions included by assigning a partial charge to the atomic sites of the surface. The resulting model is then used to study the structure of molecular arrays on the graphene and hBN surfaces, and organic molecules trapped between two layers of graphene of hBN. ${ }^{34}$

\section{Computational Methods}

In the approach used here, the atoms of the molecular environment, in this case the surface atoms, are not included in the DFT calculation but are represented by points in space that can be assigned a charge $q$. The interaction between the surface and the molecule is described by a modification of the dispersion correction to include repulsion. This is achieved through a modification of the damping function; specifically the surface-molecule interaction $E_{D I S P}$ is replaced by $E_{v d W}$, where

$$
\begin{aligned}
E_{v d W} & =-s_{6} \sum_{A}^{N_{s}} \sum_{B}^{N_{m}} \frac{C_{6}^{A B}}{R_{A B}^{6}} f_{d m p}^{r+d}\left(R_{A B}\right) \\
f_{d m p}^{r+d}\left(R_{A B}\right) & =1-e^{\left[-d\left(R_{A B} / R_{A B}^{0}-1\right)+\alpha\right]}
\end{aligned}
$$

for the $N_{s}$ surface "atoms" and $N_{m}$ molecule atoms. In this model the repulsion at short range is described by an exponential function. This functional form for the repulsion is consistent with the work of Wheatley and Price that that recognised the proportionality between the exchange-repulsion energy and the charge density overlap. ${ }^{35}$ The $R_{A B}^{0}$ values used for this contribution to the energy are derived from the experimental van der Waals radii, ${ }^{36}$ which tend to be greater than the values used in the standard DFT-D2 correction. The difference in atomic radii used for the two methods reflects the fact that in the con-

ventional approach, the DFT calculation includes interactions with substrate atoms, which will contribute to the intermolecular interaction, and this interaction is no longer present. This has also been observed in other approaches that combine empirical potentials with 
the Grimme-type dispersion correction. ${ }^{37}$ A value of $d=20.0$ is used, which is unchanged from the original damping function and $s_{6}$ and the $C_{6}^{A B}$ coefficients are also unchanged. An additional parameter $\alpha$ is introduced which allows some additional flexibility to tune the surface-molecule interaction. This parameter has values of 0 and 1.2 for graphene and hBN, respectively.

We emphasise that for the interaction between the atoms of the adsorbed molecule(s), the original, unmodified dispersion correction is used. A comparison between the original dispersion contribution, $E_{D I S P}$, and the modified function, $E_{v d W}$, is shown in Figure 1 for the interaction between a carbon atom and a nitrogen atom. The modified interaction potential replicates the behaviour of the original dispersion correction at internuclear separations greater than or equal to $R_{A B}$. However, as $R_{A B} \rightarrow R_{A B}^{0}$ the interaction becomes repulsive reflecting the interatomic repulsion at small distances. For graphene there is no charge assigned to the surface atoms, while for hBN the boron atoms have a charge of +0.85 a.u. and the nitrogen atoms a charge of -0.85 a.u, these charges were determined from a Mulliken population analysis from a calculation of the surface with no adsorbate present, although we find that the results are relatively insensitive to the value of the charge. The resulting model is termed Atomic Interactions Represented By Empirical Dispersion (AIRBED). The treatment of molecules on surfaces using a quantum mechanics/molecular mechanics (QM/MM) approach where the interaction with the surface is treated by an empirical potential has been used previously, ${ }^{38}$ however the approach presented here can be viewed as a QM/MM approach that is integrated within the DFT calculation. This feature provides the approach with the potential to be applied to a wide range of systems that would normally be studied with DFT with dispersion.

The AIRBED model is assessed through a comparison with the structure of a single molecule adsorbed on the graphene and hBN surfaces. A set of four molecules; 4,4- 


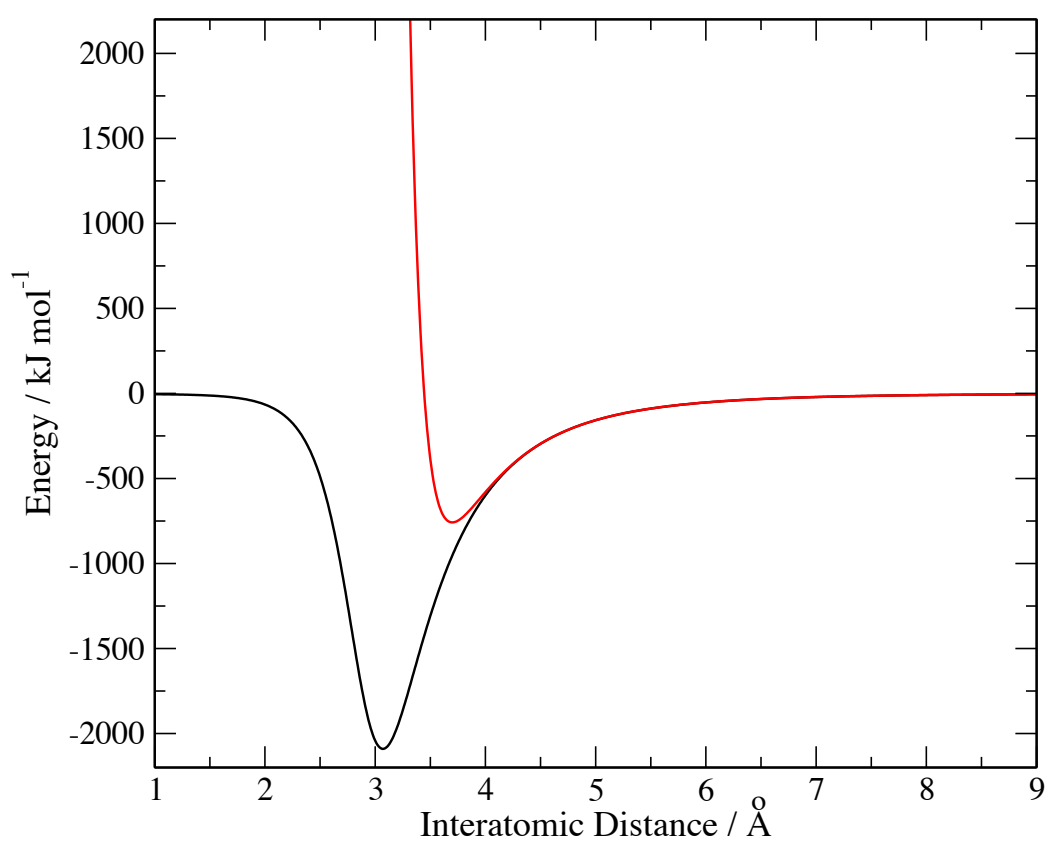

Figure 1: A comparison of the unmodified dispersion correction, $E_{D I S P}$, (black) and modified correction, $E_{v d W}$, (red) for the interaction between a carbon atom and a nitrogen atom.

diiodobiphenyl (4,4-DIB), 1,3,5-tris(4-iodophenyl)benzene (TIPB), 3,4,9,10-perylenetetracarboxylic diimide (PTCDI) and free-base phthalocyanine $\left(\mathrm{H}_{2} \mathrm{Pc}\right)$ are considered. The structure of these molecules are shown in Figure 2. These molecules include examples of planar (PTCDI and $\mathrm{H}_{2} \mathrm{Pc}$ ) and non-planar (4,4-DIB and TIPB) molecules, and are representative of molecules that have been studied on the hBN surface experimentally. Unmodified DFT-D2 calculations were performed using the B3LYP functional ${ }^{39,40}$ and 6-31G basis set. A modest basis set was used to reduce the cost of these calculations to allow larger molecules to be studied. However, for the smaller 4,4-DIB molecule it was confirmed that there is not a large dependence on the structure with respect to the larger $6-311 \mathrm{G}^{*}$ basis set and also the different $\mathrm{PBE}^{41,42}$ functional. The structure of molecules adsorbed on the surface were performed using a finite single layer model of the surface where the edge atoms are capped with hydrogen atoms derived from a B3LYP/6-31G geometry optimisation. In subsequent optimisations of surface adsorbed species, the coordinates of the surface atoms are kept fixed in position. All calculations were performed using a development version of QChem ${ }^{43}$ and visualised using 
IQmol.

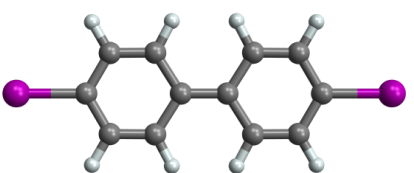

4,4-DIB

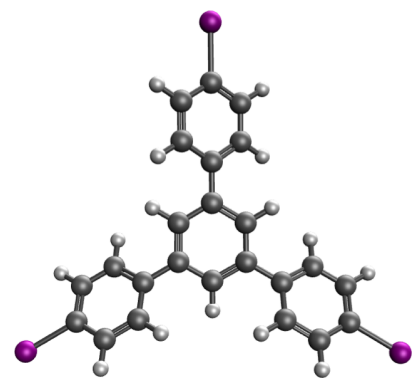

TIPB

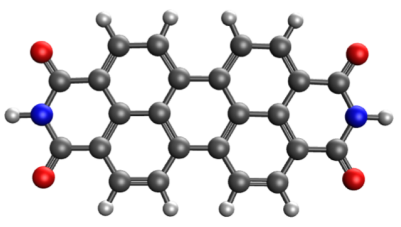

PTCDI

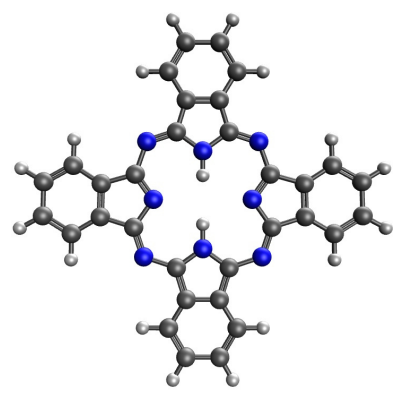

$\mathrm{H}_{2} \mathrm{Pc}$

Figure 2: Set of molecules used in study

\section{Results and Discussion}

Table 1 details a comparison between the optimised structures obtained from the full DFTD2 calculation and using the AIRBED approach. Comparison is made with the full DFT-D2 calculations because this represents the upper limit for the accuracy of the AIRBED calculations since no improvement in the underlying DFT-D2 model is made. Furthermore, determination of the structure through experiment is challenging, and for example, measurement of the height of the molecules has uncertainties that are larger than the variation between the computational values. We focus on two aspects of the structures, firstly, the height of the molecule above the surface (defined as the average height of atoms above the surface) and the root mean square deviation (RMSD) between the structures of the adsorbed molecules. The RMSD is calculated by first rotating the optimised structures using the algorithm proposed by Kabsch, ${ }^{44}$ the minimum RMSD between the structure from the full 
$\operatorname{DFT}\left(\mathbf{r}^{D F T}\right)$ and AIRBED $\left(\mathbf{r}^{A B}\right)$ calculations is evaluated according to

$$
\operatorname{RMSD}\left(\mathbf{r}^{D F T}, \mathbf{r}^{A B}\right)=\sqrt{\frac{1}{N} \sum_{i=1}^{N}\left[\left(r_{i x}^{D F T}-r_{i x}^{A B}\right)^{2}+\left(r_{i y}^{D F T}-r_{i y}^{A B}\right)^{2}+\left(r_{i z}^{D F T}-r_{i z}^{A B}\right)^{2}\right]}
$$

The DFT-D2 calculations predict that the molecules are further from the surface of graphene compared with hBN, and this trend is replicated by the AIRBED calculations. The average error in the predicted heights from AIRBED is less than $0.1 \AA$ compared with the DFT-D2 calculations, with the largest discrepancy of $0.18 \AA$ occurring for PTCDI on graphene. As expected, the RMSD for the rigid planar molecules PTCDI and $\mathrm{H}_{2} \mathrm{Pc}$ are very small and the resulting structures are indistinguishable. For the more, flexible molecules 4,4-DIB and TIPB there is an increase in the RMSD. However, an RMSD of $0.48 \AA$ still represents a close match between the structures. This is illustrated in Figure 3 which shows the DFT-D2 and AIRBED optimised structures overlaid for the two systems with largest RMSD. There are no striking visual differences but a closer inspection shows some misalignment of the phenyl rings.

Table 1: Comparison of full DFT-D2 and AIRBED calculated structures for molecules adsorbed on graphene and hBN with the B3LYP functional and 6-31G basis set. ${ }^{a}$ Root mean squared deviation between the structure of the DFT-D2 and AIRBED molecular structures.

\begin{tabular}{|l|c|c|c|}
\hline Molecule & Height DFT-D2 / $\AA$ & Height AIRBED / $\AA$ & RMSD $^{a} / \AA$ \\
\hline 4,4-DIB on graphene & 3.37 & 3.40 & 0.22 \\
4,4-DIB on hBN & 3.21 & 3.20 & 0.12 \\
PTCDI on graphene & 3.16 & 3.34 & 0.05 \\
PTCDI on hBN & 3.08 & 3.20 & 0.03 \\
TIPB on graphene & 3.42 & 3.50 & 0.19 \\
TIPB on hBN & 3.25 & 3.25 & 0.48 \\
$\mathrm{H}_{2}$ Pc on graphene & 3.22 & 3.35 & 0.04 \\
$\mathrm{H}_{2}$ Pc on hBN & 3.11 & 3.21 & 0.03 \\
\hline
\end{tabular}

Overall, the results show that the AIRBED approach provides a method for optimising the structure of molecules physisorbed on surfaces within DFT at essentially no additional computational cost to the optimisation of the structure in without the surface present. This 

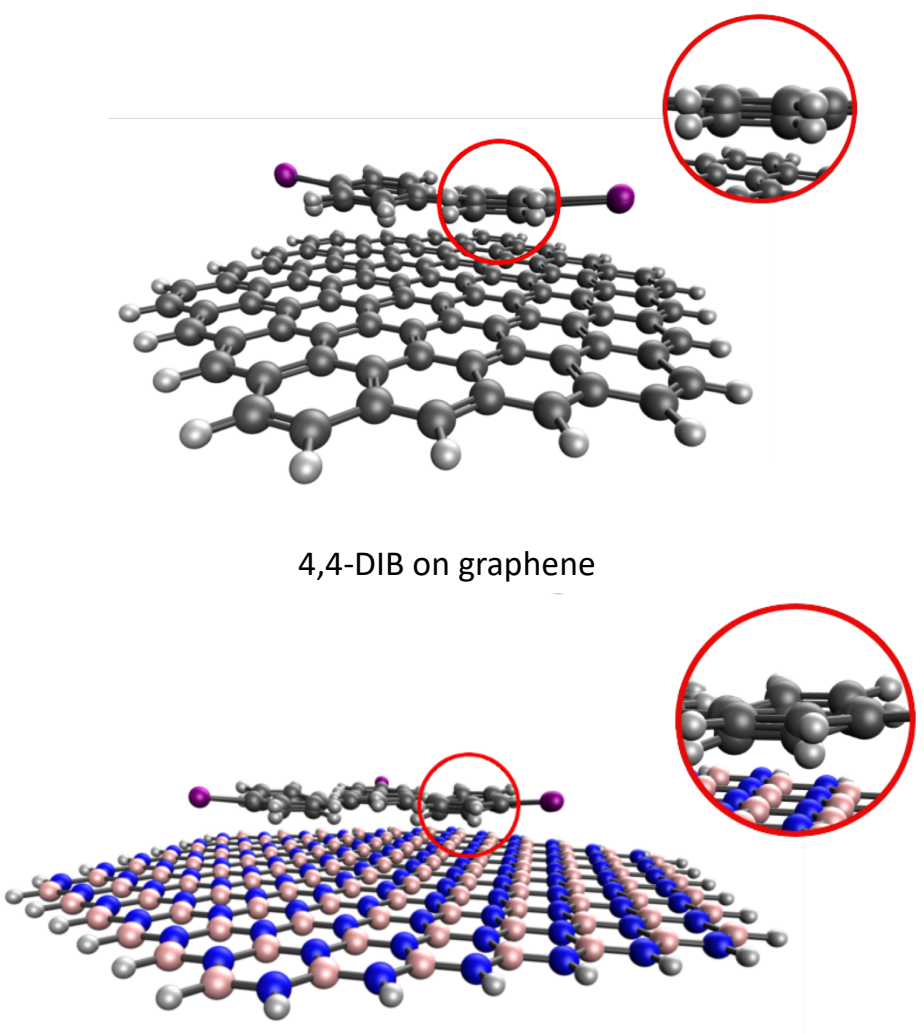

TIPB on hBN

Figure 3: A comparison between the DFT-D2 and AIRBED optimised structures for the two systems with largest RMSD. 
opens a number of potential systems and improvements to the calculations to be considered. For example, an improved description of the molecule in terms of the basis set used or much larger systems can be studied. A key interest is the structure and properties of multi-absorbate systems and molecular networks on these surfaces. ${ }^{12,18,19}$ Figure 4 shows a comparison between the relative energies of four different arrays of four $\mathrm{CO}_{2}$ molecules on the hBN surface determined by DFT-D2 and AIRBED calculations. The results show that the AIRBED model is able to predict the correct relative order of the stabilities of the different arrangements, and also the predicted relative energies are in good quantitative agreement with the full DFT-D2 calculations.

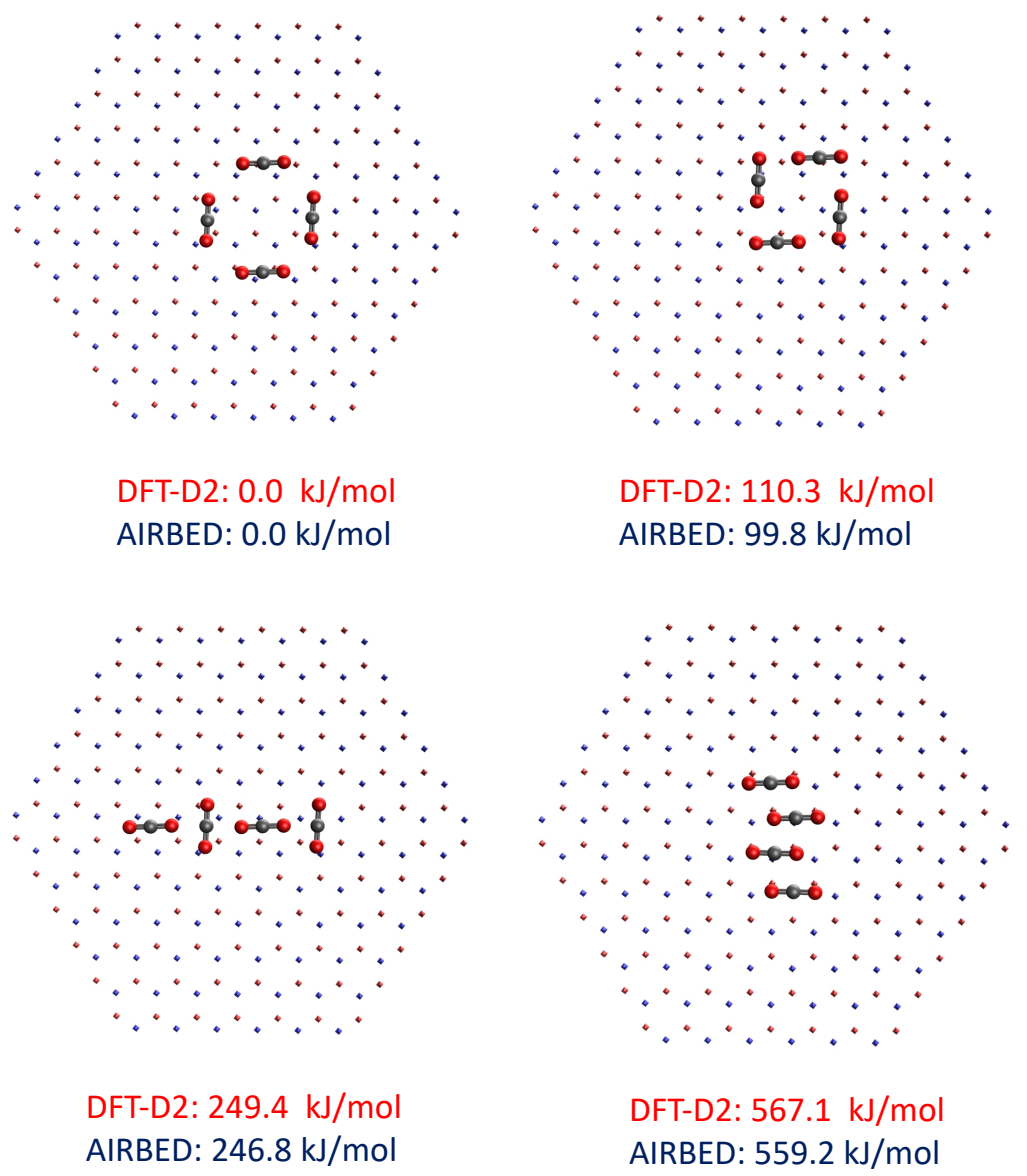

Figure 4: Comparison between the relative energies from DFT-D2 and AIRBED for arrays of four $\mathrm{CO}_{2}$ molecules on hBN. The $\mathrm{CO}_{2}$ molecules have a height of $3.02 \AA$ above the surface. 
One network that has been studied experimentally is an array of PTCDI molecules. Figure 5 illustrates the results of optimising various arrays of five PTCDI molecules. The first image shows the optimised geometry without a surface present, after starting from the experimentally observed planar structure on hBN. The resulting structure is no longer planar and has little resemblance to the observed structure of the array on the hBN surface. While for this particular molecule it is possible to retain a planar structure by imposing $\mathrm{C}_{s}$ symmetry, in general, this will not be the case. Consequently, the surface plays a vital role in the structure and organisation of the array. Figure 5 also shows the optimised structures and relative energies for five different configurations of a PTCDI array on an AIRBED hBN surface. The calculations considered a network of five PTCDI molecules which requires an AIRBED surface of over 1000 atoms. Even with relatively modest basis sets, such a calculation would be very challenging in terms of its computational cost if all of these atoms were included fully within the DFT-D2 calculation. The most stable arrangement of the array predicted by the calculations is in agreement with the array observed in experiment, with calculations predicting an average molecular spacing of $14.38 \AA$, and experiment showing spacing of $14.1 \pm$ $0.2 \AA .^{19,45,46}$ The calculations also predict that the height of the molecules of the array from the surface to be $3.15 \AA$, which is less than the corresponding distance of $3.20 \AA$ for a single molecule. The stability of this structure arises from maximising the number and strength of the hydrogen bonds and also it can be effectively extended in two-dimensions. The alternative arrays studied have fewer hydrogen bonds, and as a consequence the relative energy rises.

A further interesting system that has been studied is the 2-dimensional array of porphyrin molecules on hBN. ${ }^{18}$ Here we study four 5,10,15,20-tetrakis(4-carboxylphenyl)porphyrin (TCPP) molecules adsorbed on hBN (Figure 6). This molecule can form arrays through hydrogen bonding between the carboxylic acid groups. However, there is some conformational freedom of rotation of the phenyl groups. On adsorption on the surface there is a flattening of these rings relative to the gas-phase structure. The orientation of these rings 


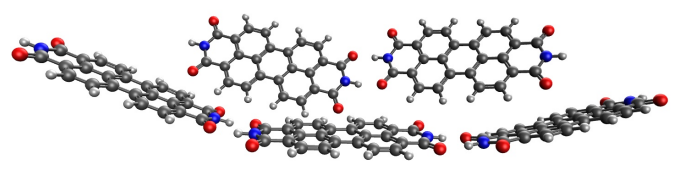

no surface

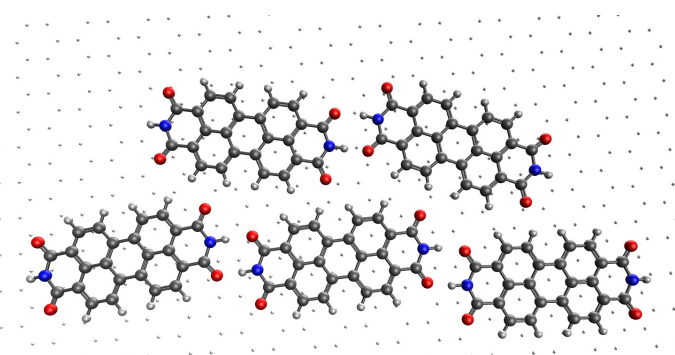

$+0.0 \mathrm{~kJ} / \mathrm{mol}$

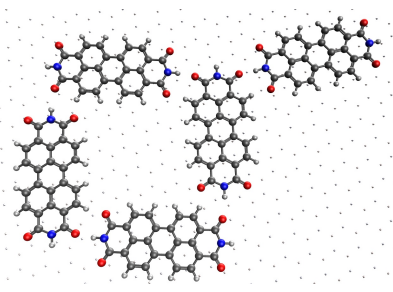

$+110.0 \mathrm{~kJ} / \mathrm{mol}$

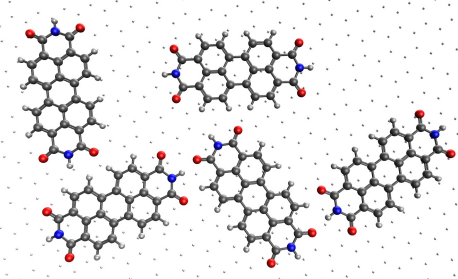

$+206.0 \mathrm{~kJ} / \mathrm{mol}$

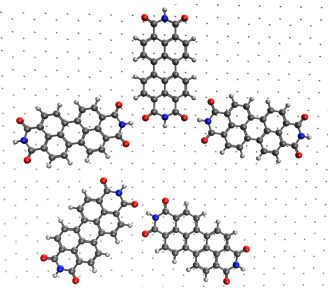

$+185.0 \mathrm{~kJ} / \mathrm{mol}$

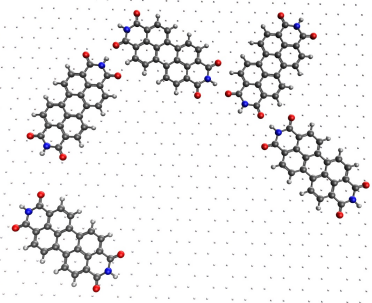

$+210.0 \mathrm{~kJ} / \mathrm{mol}$

Figure 5: The structure of an array of five PTCDI molecules optimised in gas phase and on an AIRBED surface. The relative energies and structures for five different configurations of the array on the surface are shown. 
is found to be $63^{\circ}$ for the gas-phase monomer, and $46^{\circ}$ for both the single TCPP molecule on an AIRBED surface and for the TCPP array on the AIRBED surface. For the array, the angle is evaluated from an average of the groups that are hydrogen bonded to another TCPP molecule. These calculations show a significant flattening of these groups which arises as a direct result of the surface. This flattening is also observed in full DFT-D2 calculations, ${ }^{18}$ where an angle of $50^{\circ}$ was found for a single TCPP molecule on a model hBN surface. This demonstrates that the AIRBED model of the surface is able to capture some of the subtle effects on molecular geometry caused by the surface. This is useful when considering optoelectronic properties, which can be impacted by small changes in molecular packing and conformation. ${ }^{47,48}$
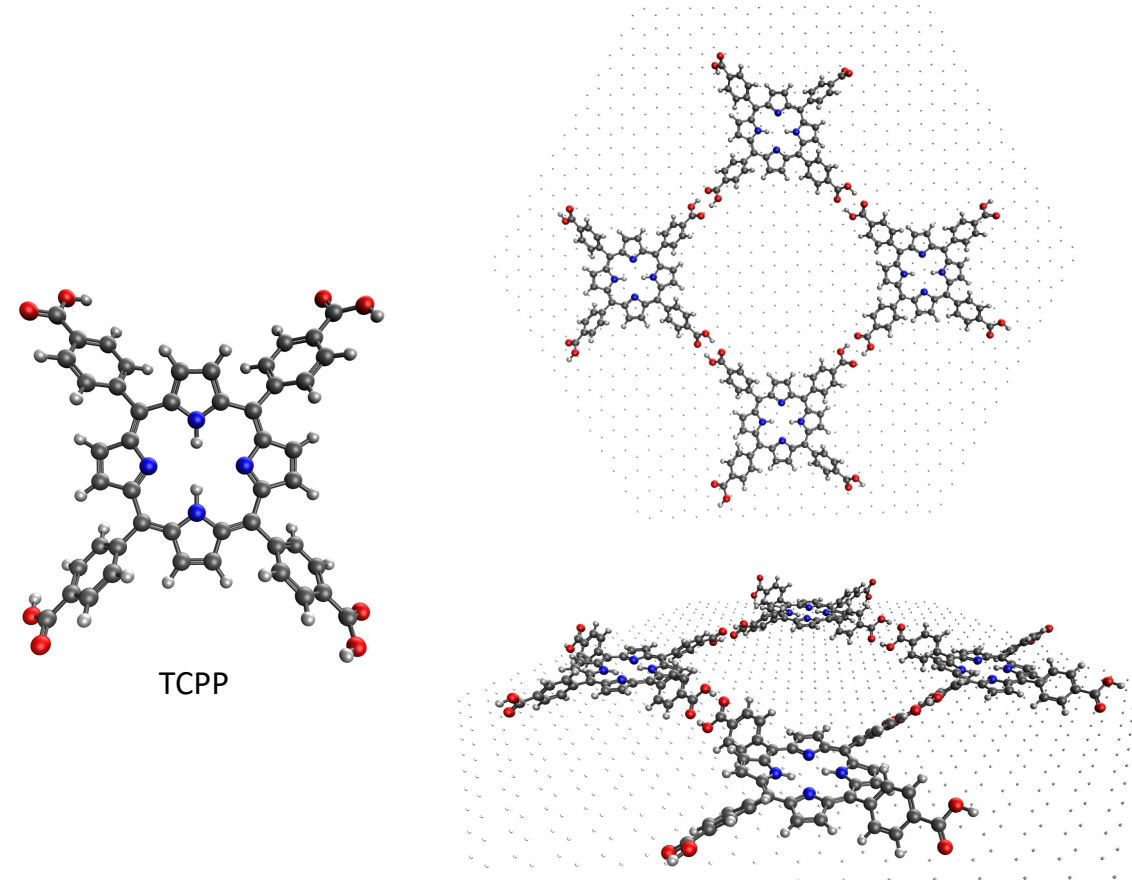

Figure 6: The structure of an array of four TCPP molecules optimised on an AIRBED hBN surface.

An extension of these systems which may be invisaged are so-called "sandwich" complexes 
where the organic layer is positioned between two layers. Within a full DFT-D2 treatment, the number of atoms required to model both layers would make the calculation very computationally demanding. However, within the AIRBED approach the inclusion of the layer does not add any practical increase in the cost of the calculation. Figure 7 shows the energy of the PTCDI molecule within two layers of graphene as the interlayer separation is varied. The lowest energy configuration occurs at a layer separation of $6.80 \AA$, where the PTCDI molecule lies in the center of the two layers. This layer spacing corresponds to about 0.1 $\AA$ larger than twice the height for a PTCDI molecule on a single layer of $3.34 \AA$. For smaller interlayer spacing the energy rises steeply. For larger separations, the PTCDI molecule does not lie at the mid point of the two layers. This is illustrated in Figure 7 for a separation of $7.25 \AA$. For this configuration there is also a noticable curvature of the PTCDI molecule which arises from the molecule maximising the dispersion interaction from both layers. As the separation increases beyond $7.25 \AA$, the molecule tends toward the configuration of a single layer and is not affected by the presence of the second layer. It is also possible to extend the model of the surface beyond a single layer. For PTCDI adsorbed on graphene, expanding the surface to include three layers showed only a small effect on the height of PTCDI molecule. For the three layer surface the height is found to be $3.33 \AA$ compared with $3.34 \AA$ for a single layer surface.

The properties of the adsorbed molecular arrays can be probed using fluorescence spectroscopy, ${ }^{18-20}$ where distinct shifts in the fluorescence bands resulting from the interaction with the surface can be measured. Simulation of fluorescence spectra requires optimisation of the structure of electronically excited states. This can be achieved within the framework of Kohn-Sham DFT by exploiting the maximum overlap method (MOM) ${ }^{49}$ which enables the treatment of excited states through constraining the orbital occupancy such that it corresponds to a desired excited state. Using this approach the structure of the $\mathrm{S}_{1}$ state of PTCDI has been optimised on the full and AIRBED surfaces. The calculated energy dif- 


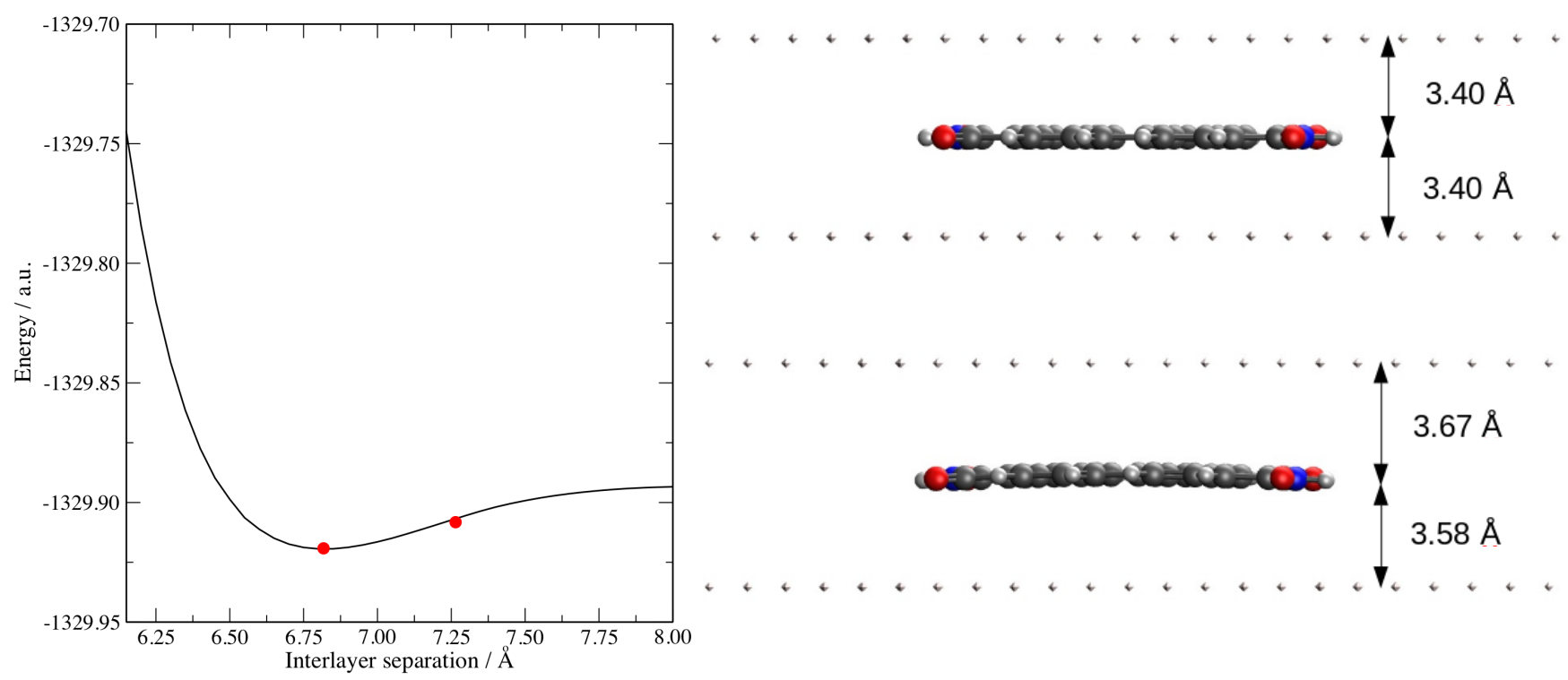

Figure 7: The energy of PTCDI between two layers of graphene with varying interlayer separation and the geometry for interlayer separations of $6.80 \AA$ and $7.25 \AA$.

ference between the $S_{0}$ and $S_{1}$ states for the two surface models agree to within $0.01 \mathrm{eV}$. This indicates that the findings for the validity of the model for ground state structures also applies for excited states.

\section{Conclusions}

A modification of the damping function used in the standard DFT-D2 method to capture repulsion in addition to dispersion interaction has been introduced. This provides a QM/MM approach integrated within the DFT calculation. The resulting AIRBED model is shown to reproduce closely the structures of molecules physisorbed on graphene and hBN surfaces predicted by full DFT-D2 calculations at a vastly reduced computational cost. The model has been used to study molecular arrays adsorbed on hBN and sandwich complexes. These systems are of interest owing to their potential uses in optoelectronic devices, however, the AIRBED approach can be applied to a much wider array of systems including the study of the molecular dynamics of the adsorbed molecules, and these are the subject on on-going 
investigations.

\section{Conflicts of interest}

There are no conflicts of interest to declare.

\section{Acknowledgements}

The authors acknowledge financial support from the UK Engineering and Physical Sciences Research Council (EP/N033906/1) and also acknowledge the University of Nottingham for the award of a studentship to SM.

\section{References}

(1) Barth, J. V.; Costantini, G.; Kern, K. Engineering atomic and molecular nanostructures at surfaces. Nature 2005, 437, 671-679.

(2) Slater, A. G.; Perdigão, L. M. A.; Beton, P. H.; Champness, N. R. Surface-based supramolecular chemistry using hydrogen bonds. Acc. Chem. Res. 2014, 47, 34173427.

(3) Mali, K. S.; Pearce, N.; De Feyter, S.; Champness, N. R. Frontiers of supramolecular chemistry at solid surfaces. Chem. Soc. Rev. 2017, 46, 2520-2542.

(4) Goronzy, D. P.; Ebrahimi, M.; Rosei, F.; Arramel,; Fang, Y.; De Feyter, S.; Tait, S. L.; Wang, C.; Beton, P. H.; Wee, A. T. S.; Weiss, P. S.; Perepichka, D. F. Supramolecular assemblies on surfaces: nanopatterning, functionality, and reactivity. ACS Nano 2018, 12, 7445-7481. 
(5) Theobald, J. A.; Oxtoby, N. S.; Phillips, M. A.; Champness, N. R.; Beton, P. H. Controlling molecular deposition and layer structure with supramolecular surface assemblies. Nature 2003, 424, 1029-1031.

(6) Stepanow, S.; Lingenfelder, M.; Dmitriev, A.; Spillmann, H.; Delvigne, E.; Lin, N.; Deng, X.; Cai, C.; Barth, J. V.; Kern, K. Steering molecular organization and hostguest interactions using two-dimensional nanoporous coordination systems. Nature Materials 2004, 3, 229-233.

(7) Grill, L.; Dyer, M.; Lafferentz, L.; Persson, M.; Peters, M. V.; Hecht, S. Nanoarchitectures by covalent assembly of molecular building blocks. Nature Nanotechnology 2007, 2, 687-691.

(8) Kühne, D.; Klappenberger, F.; Decker, R.; Schlickum, U.; Brune, H.; Klyatskaya, S.; Ruben, M.; Barth, J. V. High-Quality 2D Metal-organic coordination network providing giant cavities within mesoscale domains. J. Am. Chem. Soc. 2009, 131, 3881-3883.

(9) Gross, L.; Mohn, F.; Moll, N.; Liljeroth, P.; Meyer, G. The chemical structure of a molecule resolved by atomic force microscopy. Science 2009, 325, 1110-1114.

(10) Zhang, J.; Chen, P.; Yuan, B.; Ji, W.; Cheng, Z.; Qiu, X. Real-space identification of intermolecular bonding with atomic force microscopy. Science 2013, 342, 611-614.

(11) Sweetman, A. M.; Jarvis, S. P.; Sang, H.; Lekkas, I.; Rahe, P.; Wang, Y.; Wang, J.; Champness, N. R.; Kantorovich, L.; Moriarty, P. Mapping the force field of a hydrogenbonded assembly. Nature Communications 2014, 5, 3931.

(12) Korolkov, V. V.; Svatek, S. A.; Allen, S.; Roberts, C. J.; Tendler, S. J. B.; Taniguchi, T.; Watanabe, K.; Champness, N. R.; Beton, P. H. Bimolecular porous supramolecular networks deposited from solution on layered materials: graphite, boron nitride and molybdenum disulphide. Chem. Commun. 2014, 50, 8882-8885. 
(13) Auwärter, W. Hexagonal boron nitride monolayers on metal supports: Versatile templates for atoms, molecules and nanostructures. Surf. Sci. Rep. 2019, 74, $1-95$.

(14) Müller, M.; Langner, A.; Krylova, O.; Le Moal, E.; Sokolowski, M. Fluorescence spectroscopy of ultrathin molecular organic films on surfaces. Appl. Phys. B 2011, 105, 67.

(15) Müller, M.; Le Moal, E.; Scholz, R.; Sokolowski, M. Exciton and polarization contributions to optical transition energies in an epitaxial organic monolayer on a dielectric substrate. Phys. Rev. B 2011, 83, 241203.

(16) Müller, M.; Paulheim, A.; Eisfeld, A.; Sokolowski, M. Finite size line broadening and superradiance of optical transitions in two dimensional long-range ordered molecular aggregates. J. Chem. Phys. 2013, 139, 044302.

(17) Vlaming, S. M.; Eisfeld, A. Tunable superradiance in porphyrin chains on insulating surfaces. J. Phys. D 2014, 47, 305301.

(18) Korolkov, V. V.; Svatek, S. A.; Summerfield, A.; Kerfoot, J.; Yang, L.; Taniguchi, T.; Watanabe, K.; Champness, N. R.; Besley, N. A.; Beton, P. H. van der Waals-induced chromatic shifts in hydrogen-bonded two-dimensional porphyrin arrays on boron nitride. ACS Nano 2015, 9, 10347-10355.

(19) Kerfoot, J.; Korolkov, V. V.; Nizovtsev, A. S.; Jones, R.; Taniguchi, T.; Watanabe, K.; Lesanovsky, I.; Olmos, B.; Besley, N. A.; Besley, E.; Beton, P. H. Substrate-induced shifts and screening in the fluorescence spectra of supramolecular adsorbed organic monolayers. J. Chem. Phys, 2018, 149, 054701.

(20) Alkhamisi, M.; Korolkov, V. V.; Nizovtsev, A. S.; Kerfoot, J.; Taniguchi, T.; Watanabe, K.; Besley, N. A.; Besley, E.; Beton, P. H. The growth and fluorescence of phthalocyanine monolayers, thin films and multilayers on hexagonal boron nitride. Chem. Comm. 2018, 54, 12021-12024. 
(21) Lazar, P.; Karlický, F.; Jurečka, P.; Kocman, M.; Otyepková, E.; Šafářová, K.; Otyepka, M. Adsorption of small organic molecules on graphene. J. Am. Chem. Soc. 2013, 135, 6372-6377.

(22) Karlický, F.; Otyepková, E.; Banáš, P.; Lazar, P.; Kocman, M.; Otyepka, M. Interplay between ethanol adsorption to high-energy sites and clustering on graphene and graphite alters the measured isosteric adsorption enthalpies. J. Phys. Chem. C 2015, 119, 20535-20543.

(23) Karlický, F.; Otyepková, E.; Lo, R.; Pitoňák, M.; Jurečka, P.; Pykal, M.; Hobza, P.; Otyepka, M. Adsorption of organic molecules to van der Waals materials: Comparison of fluorographene and fluorographite with graphene and graphite. J. Chem. Theory Computat. 2017, 13, 1328-1340.

(24) Grimme, S.; Antony, J.; Schwabe, T.; Mück-Lichtenfeld, C. Density functional theory with dispersion corrections for supramolecular structures, aggregates, and complexes of (bio)organic molecules. Org. Biomol. Chem. 2007, 5, 741-758.

(25) Foster, M. E.; Sohlberg, K. Empirically corrected DFT and semi-empirical methods for non-bonding interactions. Phys. Chem. Chem. Phys. 2010, 12, 307-322.

(26) Grimme, S. Density functional theory with London dispersion corrections. Wiley Interdisciplinary Reviews: Computational Molecular Science 2011, 1, 211-228.

(27) Grimme, S. Accurate description of van der Waals complexes by density functional theory including empirical corrections. J. Comp. Chem. 2004, 25, 1463-1473.

(28) Grimme, S. Semiempirical GGA-type density functional constructed with a long-range dispersion correction. J. Comp. Chem. 2006, 27, 1787-1799.

(29) Dion, M.; Rydberg, H.; Schröder, E.; Langreth, D. C.; Lundqvist, B. I. Van der Waals density functional for general geometries. Phys. Rev. Lett. 2004, 92, 246401. 
(30) Becke, A. D.; Johnson, E. R. Exchange-hole dipole moment and the dispersion interaction. J. Chem. Phys. 2005, 122, 154104.

(31) Vydrov, O. A.; Van Voorhis, T. Nonlocal van der Waals density functional made simple. Phys. Rev. Lett. 2009, 103, 063004.

(32) Tkatchenko, A.; Scheffler, M. Accurate molecular van der Waals interactions from ground-state electron density and free-atom reference data. Phys. Rev. Lett. 2009, 102, 073005.

(33) Briggs, E. A.; Besley, N. A. Modelling excited states of weakly bound complexes with density functional theory. Phys. Chem. Chem. Phys. 2014, 16, 14455-14462.

(34) Vasu, K. S.; Prestat, E.; Abraham, J.; Dix, J.; Kashtiban, R. J.; Beheshtian, J.; Sloan, J.; Carbone, P.; Neek-Amal, M.; Haigh, S. J.; Geim, A. K.; Nair, R. R. Van der Waals pressure and its effect on trapped interlayer molecules. Nature Communications 2016, 7, 12168.

(35) Wheatley, R. J.; Price, S. L. An overlap model for estimating the anisotropy of repulsion. Mol. Phys. 1990, 69, 507-533.

(36) Bondi, A. van der Waals volumes and radii. J. Phys. Chem. 1964, 68, 441-451.

(37) Tailor, P. M.; Wheatley, R. J.; Besley, N. A. Simulation of the Raman spectroscopy of multi-layered carbon nanomaterials. Phys. Chem. Chem. Phys. 2018, 20, 28001-28010.

(38) Floss, G.; Granucci, G.; Saalfrank, P. Surface hopping dynamics of direct trans $\rightarrow$ cis photoswitching of an azobenzene derivative in constrained adsorbate geometries. $J$. Chem. Phys. 2012, 137, 234701.

(39) Becke, A. D. A new mixing of Hartree-Fock and local density-functional theories. J. Chem. Phys. 1993, 98, 1372-1377. 
(40) Stephens, P. J.; Devlin, F. J.; Chabalowski, C. F.; Frisch, M. J. Ab initio calculation of vibrational absorption and circular dichroism spectra using density functional force fields. J. Phys. Chem. 1994, 98, 11623-11627.

(41) Perdew, J. P.; Burke, K.; Ernzerhof, M. Generalized gradient approximation made simple. Phys. Rev. Lett. 1996, 77, 3865-3868.

(42) Perdew, J. P.; Burke, K.; Ernzerhof, M. Generalized gradient approximation made simple [Phys. Rev. Lett. 77, 3865 (1996)]. Phys. Rev. Lett. 1997, 78, 1396-1396.

(43) Shao, Y. et al. Advances in molecular quantum chemistry contained in the Q-Chem 4 program package. Mol. Phys. 2015, 113, 184-215.

(44) Kabsch, W. A solution for the best rotation to relate two sets of vectors. Acta Crystallographica Section A 1976, 32, 922-923.

(45) Swarbrick, J. C.; Ma, J.; Theobald, J. A.; Oxtoby, N. S.; O'Shea, J. N.; Champness, N. R.; Beton, P. H. Square, hexagonal, and row phases of PTCDA and PTCDI on $\mathrm{Ag}-\mathrm{Si}(111) \times R 30^{\circ}$. J. Phys. Chem. B 2005, 109, 12167-12174.

(46) Mura, M.; Silly, F.; Briggs, G. A. D.; Castell, M. R.; Kantorovich, L. N. H-Bonding supramolecular assemblies of PTCDI molecules on the $\mathrm{Au}(111)$ surface. J. Phys. Chem. C 2009, 113, 21840-21848.

(47) Hasegawa, T.; Ashizawa, M.; Matsumoto, H. Design and structure-property relationship of benzothienoisoindigo in organic field effect transistors. RSC Adv. 2015, 5, 61035-61043.

(48) Wang, C.; Li, Z. Molecular conformation and packing: their critical roles in the emission performance of mechanochromic fluorescence materials. Mater. Chem. Front. 2017, 1, 2174-2194. 
(49) Gilbert, A. T. B.; Besley, N. A.; Gill, P. M. W. Self-consistent field calculations of excited states using the maximum overlap method (MOM). J. Phys. Chem. A 2008, 112, 13164-13171. 